\title{
Distribution patterns of demyelination correlate with clinical profiles in chronic inflammatory demyelinating polyneuropathy
}

\author{
S Kuwabara, K Ogawara, S Misawa, M Mori, T Hattori
}

See end of article for authors' affiliations

.....................

Correspondence to: Dr S Kuwabara,

Department of Neurology, Chiba University School of Medicine, 1-8-1 Inohana,

Chuo-ku, Chiba

260-8670, Japan;

kuwabara@

med.m.chiba-u.ac.jp

Received 1 March 2001 In final revised form 9 August 2001

Accepted 17 August 2001
Background: Chronic inflammatory demyelinating polyneuropathy (CIDP) is a heterogeneous disorder having a wide clinical range, and is characterised by multifocal demyelination that can involve the distal nerve terminals, intermediate nerve segments, and nerve roots.

Objective: To investigate whether the distribution patterns of demyelination along the course of the nerve correlate with clinical profiles in patients with CIDP.

Methods: Motor nerve conduction studies were carried out on 42 consecutive patients. According to the physiological criteria for demyelination, the presence of a demyelinative lesion was determined in the distal nerve segments (distal pattern) or intermediate nerve segments (intermediate pattern), or in both (diffuse pattern). The serum concentration of tumour necrosis factor (TNF)- $\alpha$ was measured by immunoassay.

Results: Patients were classified as having a distal $(n=10)$, intermediate $(n=13)$, or diffuse $(n=15)$ pattern, or were unclassified $(n=4)$. Patients with the distal or diffuse pattern had common clinical features such as subacute onset, symmetric symptoms, and weakness involving proximal as well as distal muscles. Patients with the distal pattern had a good response to treatment and a monophasic remitting course, but the diffuse pattern was associated with a treatment dependent relapsing course, reflecting longer disease activity. The serum TNF- $\alpha$ concentrations increased only in the "diffuse" subgroup of patients, and this might be associated with breakdown of the blood-nerve barrier and therefore, involvement of the intermediate segments. The intermediate pattern was characterised by a chronic course, asymmetric symptoms, less severe disability, and refractoriness to treatments.

Conclusions: CIDP consists of subtypes with varying predilections for lesions along the course of the nerve. The distribution patterns of conduction abnormalities may be useful in the prediction of outcome of patients with CIDP.

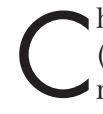
hronic inflammatory demyelinating polyneuropathy (CIDP) is a diagnostic term dependent on pattern recognition, and is based on clinical symptoms and signs, electrodiagnostic studies, CSF examination, and other laboratory tests. ${ }^{1}$ It is likely that CIDP is a heterogeneous disorder, ${ }^{2}$ having a wide range of clinical expression ranging from subacute ${ }^{3}$ to chronic progression, and a monophasic to a relapsing course. ${ }^{245}$ In addition, it can include predominantly proximal to distal weakness, ${ }^{6}$ and may involve neuropathies ranging from symmetric polyneuropathy to mononeuropathy multiplex..$^{78}$

The disorder usually presents as a more or less symmetric sensorimotor neuropathy, ${ }^{2}$ but many cases with obviously asymmetric symptoms have been described as variants of CIDP. $^{7-10}$ Moreover, the criteria formulated by the ad hoc subcommittee of the American Academy of Neurology AIDS task force, ${ }^{1}$ which have been the most widely used criteria for the diagnosis of CIDP, do not require symmetric symptoms.

Multifocal demyelination is a diagnostic hallmark of CIDP, but distribution of demyelinative lesions varies among patients. Postmortem studies have often shown demyelinative lesions dominant in the nerve roots. ${ }^{11}$ The distal nerve terminals may be preferentially affected by CIDP: after successful treatment, patients show an obvious increase in the amplitude of distally evoked compound muscle action potential, suggesting resolution of distal conduction block. ${ }^{12}$ Moreover, some patients, especially with an asymmetric variant of CIDP, often show conduction block localised in the intermediate nerve segment. ${ }^{78}$
We performed a retrospective analysis of our patients to determine whether the distribution patterns of nerve conduction abnormalities are associated with clinical features and response to immunomodulating treatment. We discuss the utility of this approach based on distribution patterns of demyelinative lesions, as opposed to the more common distinction that emphasises the presence or absence of serum $\mathrm{M}$ proteins or antibodies directed against peripheral nerves.

\section{PATIENTS AND METHODS \\ Patients}

Clinical and electrophysiological data were reviewed retrospectively for 42 consecutive patients seen at Chiba University Hospital between 1991 and 2000. Their condition fulfilled the research criteria for diagnosis of CIDP. ${ }^{1}$ We excluded patients with monoclonal gammopathy or multifocal motor neuropathy, because their clinical and immunological profiles and response to treatment have been shown to be somewhat different from those of idiopathic CIDP. ${ }^{13-16}$ A functional assessment was performed using the Hughes functional grading scale: 0 , normal; 1 , able to run with minor symptoms and signs; 2 , able to walk 5 meters independently; 3 , able to walk 5 meters with aids; 4 , chair or bedbound. We focused on the

Abbreviations: CIDP, chronic inflammatory demyelinating polyneuropathy; TNF, tumour necrosis factor; DL, distal latency; CV, conduction velocity; TLls, terminal latency indices; AMNSSR, abnormal median-normal sural sensory nerve response 
asymmetry of symptoms, which was defined as differences in muscle strength by one or more MRC scales in the homonymous muscles.

\section{Treatments}

Thirty four patients were initially treated with high dose corticosteroids: 28 patients received intravenous methylprednisolone pulse therapy ( $1000 \mathrm{mg} /$ day for 3 days) which was followed by oral administration of $60 \mathrm{mg}$ prednisolone daily or $100 \mathrm{mg}$ on alternate days, for 4 to 6 weeks. In the remaining six patients, treatment was started with $60 \mathrm{mg}$ prednisolone for 4 to 8 weeks. The dosage of prednisolone was gradually reduced by $5 \mathrm{mg} / \mathrm{month}$. Fifteen patients, including those who did not respond to corticosteroid treatment, were treated with plasmapheresis or intravenous immunoglobulin infusion. Eight patients received no immunomodulating treatment because of the mildness of their neurological disability. Treatment was considered effective when the patient's condition improved by one or more on the Hughes grade.

\section{Electrophysiology}

Motor nerve conduction studies were performed in the median, ulnar, tibial, and peroneal nerves using conventional procedures. Antidromic sensory nerve conduction studies were performed in the median and sural nerves. According to the electrodiagnostic criteria for demyelination, ${ }^{1}$ the presence of demyelinative conduction abnormalities of the median and ulnar nerves on the right was determined in the distal nerve segments (at the wrist) or intermediate nerve segments (wrist to elbow), or in both. Patients were classified as having "distal" demyelination when distal latencies were $>125 \%$ of the upper limit of normal, or as having "intermediate" demyelination when conduction velocities were $<80 \%$ of lower limits of normal, or there was conduction block/abnormal temporal dispersion ( $>20 \%$ drop in the negative area of compound muscle action potential). Conduction block and abnormal temporal dispersion were not distinguished because temporal dispersion can cause a decrease in area of compound muscle action potential up to $50 \% .{ }^{17}$ When there were demyelinative conduction abnormalities in both distal and intermediate segments, patients were classified as having " diffuse" demyelination. When distribution patterns were not consistent between the median and ulnar nerves, patients were regarded as unclassified. F waves were recorded after wrist stimulation.

Terminal latency index was used to compare the distal segment (distal to the wrist) with the intermediate segment (wrist to elbow). It was calculated using the formula developed by Shahani et $a^{18}$ :

Distal conduction distance $(\mathrm{mm})$ /forearm conduction velocity $(\mathrm{m} / \mathrm{s}) /$ distal latency ( $\mathrm{ms}$ )

Distal conduction distance was measured from the recording electrode over the muscle to the site of wrist stimulation.

In sensory nerve conduction studies, we focused on the involvement patterns of the median and sural sensory nerve responses. Median nerve studies examined the most distal part of the nerve, whereas the intermediate portion was tested in sural nerve studies. When demyelination occurs dominantly in the distal nerve terminals, median sensory response would be affected more severely, whereas sural response would remain normal. This pattern of involvement has been reported as "abnormal median-normal sural sensory response", and is specifically seen in patients with CIDP or Guillain-Barré syndrome. ${ }^{19}$

\section{Serum tumour necrosis factor- $\alpha$ assays}

Concentrations of serum TNF- $\alpha$ were determined by an immunoassay (AN'ALYZA ${ }^{\mathrm{TM}}$, human TNF- $\alpha$, Genzyme Techne, Minneapolis, USA). The test serum was added to the well of an enzyme linked immunosorbent assay (ELISA) plate which had been preincubated with monoclonal antibody to recom- binant human TNF- $\alpha$. A standard curve was run on each assay plate using recombinant human TNF- $\alpha$ in serial dilutions. If concentrations were below $4.4 \mathrm{pg} / \mathrm{ml}$, the values were replaced by $4.4 \mathrm{pg} / \mathrm{ml}$ because the minimum detectable dose of TNF- $\alpha$ in this assay was less than $4.4 \mathrm{pg} / \mathrm{ml}$. Concentrations were regarded as increased if they were higher than 3SD above the mean value of 49 normal control samples.

\section{Statistical analysis}

Differences in proportions were tested by $\chi^{2}$ or Fisher's exact test, and differences in medians with the Mann-Whitney test, using Stat View 4.5 software.

\section{RESULTS}

Forty two patients ( 25 men and 17 women) were diagnosed as having CIDP over the study period. They ranged in age from 14 to 79 years (mean 48 years). The duration of their neurological symptoms before our first examination ranged from 2 to 144 months (mean 18 months). The average time of follow up was 53 months with a range of 12 to 119 months.

\section{Distribution patterns of demyelinative nerve conduction abnormalities}

Table 1 shows the numbers of patients classified into each category according to results of median and ulnar motor nerve conduction studies. Most of the patients showed concordance between the electrophysiological classification in the two nerves. Thirty eight patients were classified as having the "distal" $(\mathrm{n}=10)$, "intermediate" $(\mathrm{n}=13)$, or "diffuse" $(\mathrm{n}=15)$ pattern. The remaining four patients were unclassifiable because results for the two nerves were inconsistent. Representative cases in each category are shown in figure 1. Data of $\mathrm{F}$ wave studies were not analyzed because they were absent or unidentified due to contamination of A waves in 17 $(45 \%)$ of the 38 patients. One of the four unclassified patients showed $\mathrm{F}$ wave absence as an isolated conduction abnormality. Results of tibial and peroneal nerve studies were not analyzed because compound muscle action potentials were not elicited in $10(24 \%)$ of the 42 patients.

Results of terminal latency indexes (TLIs) were used to confirm the electrophysiological classification. Patients with the distal pattern had significantly smaller TLI $(0.15$ (SD $0.005)$; $<<0.01)$, compared with patients with the intermediate $(0.41$ (SD 0.07)) or diffuse $(0.27$ (SD 0.12)) pattern, and normal subjects (0.31 (SD 0.04)), suggestive of the presence of prominent conduction slowing in the distal nerve segments. ${ }^{18}$ By contrast, patients with the intermediate pattern showed significantly greater TLI $(\mathrm{p}<0.01)$, suggesting that conduction slowing was predominant in the forearm segments. The following analyses were made on 38 patients who were classified into one of the three categories.

Table 1 Numbers of patients calssified into each category according to nerve conduction study results

\begin{tabular}{lllll}
\hline & \multicolumn{3}{l}{ Ulnar nerve } \\
\cline { 2 - 5 } & $\begin{array}{l}\text { Distal } \\
(n=8)\end{array}$ & $\begin{array}{l}\text { Intermediate } \\
(n=15)\end{array}$ & $\begin{array}{l}\text { Diffuse } \\
(n=13)\end{array}$ & $\begin{array}{l}\text { Others } \\
(n=7)\end{array}$ \\
\hline Median nerve: & 7 & 0 & 0 & 3 \\
Distal $(n=10)$ & 0 & 11 & 0 & 1 \\
Intermediate $(n=12)$ & 0 & 2 & 12 & 2 \\
Diffuse $(n=17)$ & 1 & 1 & 1 & 1 \\
Others $(n=3)$ & 0 & 1 &
\end{tabular}

Distal, demyelinative abnormalities restricted to the distal nerve segments; intermediate, demyelinative abnormalities restricted to the intermediate nerve segments; diffuse, demyelinative abnormalities in both the distal and intermediate segments; others, criteria not met for demyelination in both the distal and intermediate segments. 

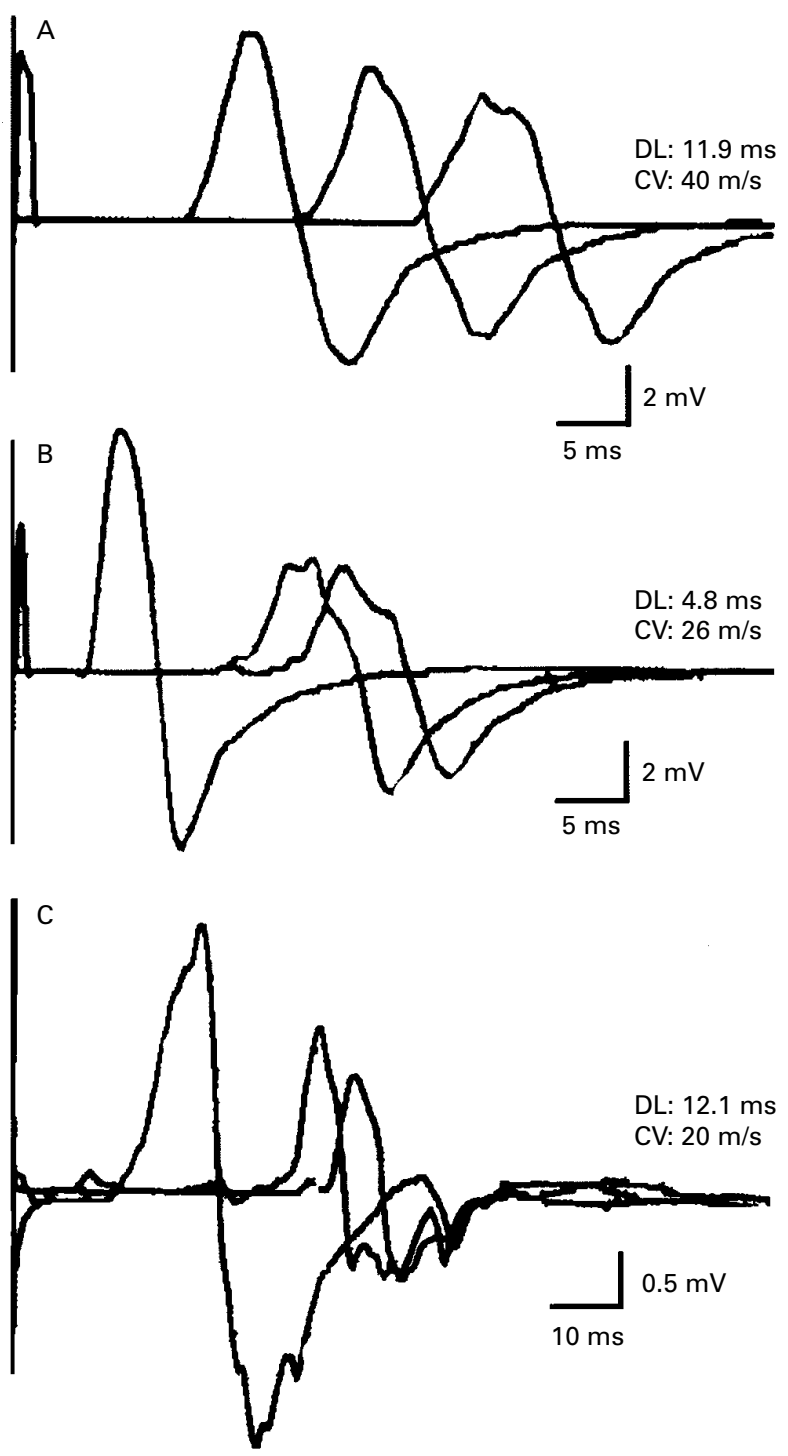

Figure 1 Representative findings of median motor nerve conduction studies in each category. Compound muscle action potentials are recorded from the abductor pollicis brevis with stimulation sites at the wrist, elbow, and axilla. According to the criteria for demyelination, cut off values are $5.7 \mathrm{~ms}$ for distal latency (DL), and $37 \mathrm{~m} / \mathrm{s}$ for conduction velocity (CV). (A) Distal pattern: DL is disproportionally prolonged. (B) Intermediate pattern: slowed CV and abnormal temporal dispersion between the wrist and elbow. (C) Diffuse pattern: both $\mathrm{DL}$ and $\mathrm{CV}$ are in the range of demyelination, and conduction block is present between the wrist and elbow.

\section{Clinical and laboratory features}

Among the three patient groups, age of onset and male to female ratio did not differ significantly. A number of clinical profiles were significantly different among the patient groups. Duration of illness before our first examination was significantly longer for patients with the intermediate pattern $(42$ (SD 55) months), compared with those with the distal (7 (SD 9) months) or diffuse (8 (SD 7) months) pattern. Almost $(96 \%)$ all of the patients with the distal or diffuse pattern had symmetric polyneuropathy, whereas $60 \%$ of them showed weakness involving the proximal as well as distal muscles. By contrast, asymmetry of muscle weakness was found in nine $(69 \%)$ of the 13 patients with the intermediate pattern: only one patient had typical mononeuropathy multiplex, and the remaining eight had generalised but asymmetric polyneuropathy. The "diffuse" group of patients showed a significantly higher Hughes grade than did the other two groups of patients. The concentration of CSF protein was higher in the "distal" (122 (SD 95) mg/dl) and "diffuse" (148 (SD 138) $\mathrm{mg} / \mathrm{dl})$ groups, compared with the "intermediate" group (80 (SD 46) mg/dl; p<0.05).

For TNF- $\alpha$ assays, serum samples before immune treatment were available in 22 patients who had the distal $(n=8)$, intermediate $(n=7)$, or diffuse $(n=7)$ pattern. In 49 normal subjects, the mean (SD) serum TNF- $\alpha$ concentration was 8.8 (5.7) $\mathrm{pg} / \mathrm{ml}$. The cut off value (mean (3SD) of normal samples) was therefore set to $25.9 \mathrm{pg} / \mathrm{ml}$. Raised serum concentrations of TNF- $\alpha$ were detected in five ( $23 \%$ ) of the 22 patients, and all five had the diffuse pattern. The mean value of serum concentrations of TNF- $\alpha$ was markedly higher in patients with the diffuse pattern (39.1 (SD 10.1) pg/ml; p<0.01), compared with patients with the distal $(6.1$ (SD 1.2) pg/ml) or intermediate $(7.5$ (SD 1.9) $\mathrm{pg} / \mathrm{ml}$ ) pattern, and normal subjects $(8.8$ (SD 5.7) $\mathrm{pg} / \mathrm{ml}$ ).

\section{Response to treatments, and outcomes}

Table 2 shows the relation of the electrodiagnosis pattern with response to treatment or a clinical course. Patients with the distal pattern showed good responses to corticosteroid treatment, and a monophasic remitting course. The "intermediate" group patients were relatively refractory to treatment with steroids or plasmapheresis, and tended to have chronic progressive or stable courses. Patients with the "diffuse" pattern often showed improvement after immunomodulating therapies, but often had relapsing courses, and almost all of their relapses occurred after stopping therapy (treatment dependent relapse).

At the end of follow up (mean (range) 53 (12 to 119) months after the initiation of treatment or entry), 18 (47\%) of the 38 patients had had long lasting remissions (more than 12 months after treatment stopped): nine of them had the distal pattern, three had the intermediate pattern, and six had the diffuse pattern. Nine $(90 \%)$ of the 10 patients with the distal

\begin{tabular}{|c|c|c|c|c|}
\hline \multicolumn{5}{|c|}{ on of electrodiagnosis with response to treatment and clinical } \\
\hline & \multicolumn{3}{|c|}{ Electrodiagnosis } & \multirow[b]{2}{*}{$\mathrm{p}$ Value } \\
\hline & $\begin{array}{l}\text { A: distal } \\
(n=10)(\%)\end{array}$ & $\begin{array}{l}\text { B: intermediate } \\
(n=13)(\%)\end{array}$ & $\begin{array}{l}\text { C: diffuse } \\
(n=15)(\%)\end{array}$ & \\
\hline \multicolumn{5}{|c|}{ Response to treatment: } \\
\hline Corticosteroid & $7 / 7(100)$ & $3 / 9(33)$ & $7 / 14(50)$ & $0.01 *, 0.03 \dagger$ \\
\hline Plasmapheresis & $2 / 2(100)$ & $1 / 4(25)$ & $4 / 7(57)$ & NS \\
\hline Immunoglobulin & $1 / 1(100)$ & $4 / 4(100)$ & $4 / 4(100)$ & NS \\
\hline \multicolumn{5}{|l|}{ Course: } \\
\hline Monophasic & $8(80)$ & $0(0)$ & $6(40)$ & $0.00009^{*}$ \\
\hline Relapsing & $2(20)$ & $5(38)$ & $9(60)$ & \\
\hline Chronic & $0(0)$ & $8(62)$ & $0(0)$ & $0.003 *, 0.0006 \ddagger$ \\
\hline
\end{tabular}


Table 3 Correlation of electrodiagnosis with outcome

\begin{tabular}{|c|c|c|c|c|}
\hline & \multicolumn{3}{|c|}{ Electrodiagnosis } & \multirow[b]{2}{*}{$\mathrm{p}$ Value } \\
\hline & $\begin{array}{l}\text { A: distal } \\
(n=10)\end{array}$ & $\begin{array}{l}\text { B: intermediate } \\
(n=13)\end{array}$ & $\begin{array}{l}\text { C: diffuse } \\
(n=15)\end{array}$ & \\
\hline \multicolumn{5}{|l|}{ Hughes grade: mean (range): } \\
\hline Before treatment & $2.4(1-4)$ & $2.0(1-4)$ & $3.5(2-4)$ & $0.005 \dagger, 0.00004 \ddagger$ \\
\hline Best during a course & $1.2(0-2)$ & $1.6(1-4)$ & $2.3(0-4)$ & NS \\
\hline At the end of follow up & $1.2(0-2)$ & $1.8(1-4)$ & $2.9(1-4)$ & $0.007^{*}, 0.02 \ddagger$ \\
\hline
\end{tabular}

Table 4 Relation of sensory nerve conduction with motor nerve electrodiagnosis and outcome

\begin{tabular}{llll}
\hline & \multicolumn{3}{l}{$\begin{array}{l}\text { Abnormal } \\
\text { median-normal sural } \\
\text { sensory response }\end{array}$} \\
\cline { 2 - 3 } & $\begin{array}{c}\text { Present } \\
(n=14)\end{array}$ & $\begin{array}{c}\text { Absent } \\
(n=24)\end{array}$ & $\mathrm{p}$ Value \\
\hline $\begin{array}{c}\text { Motor electrodiagnosis: } \\
\text { Distal }(n=10)\end{array}$ & 9 & 1 & 0.00005 \\
$\begin{array}{l}\text { Intermediate }(n=13) \\
\text { Diffuse }(n=15)\end{array}$ & 0 & 13 & 0.0007 \\
$\begin{array}{c}\text { Outcome: } \\
\text { Long lasting remission* }(n=18)\end{array}$ & 12 & 10 & NS \\
$\begin{array}{c}\text { Treatment dependent relapsing } \\
\text { course }(n=12)\end{array}$ & 2 & 10 & 0.001 \\
Chronic progressive/stable (n=8) & 0 & 8 & 0.03 \\
\hline *Lasting for $>12$ months after stopping immune treatment. \\
\hline
\end{tabular}

pattern eventually had long lasting remissions for a mean follow up period of 58 months (range 12 to 119 months). Patients with the intermediate pattern showed some response to immunoglobulin therapy, but the overall course was chronic progressive or chronic stable in most of them. Table 3 shows correlation of electrodiagnosis with outcome. Before treatment, the diffuse pattern was associated with more severe disabilities than the other patterns. At the end of follow up, all 10 patients with the distal pattern were able to walk (Hughes grades 0 to 2), whereas two (15\%) of the 13 patients with the intermediate pattern and four (27\%) of the 15 with the diffuse pattern had not regained the ability to walk independently.

\section{Patterns of sensory nerve conduction, and outcomes}

Abnormal median-normal sural sensory nerve response $(\text { AMNSSR })^{19}$ was found for $14(37 \%)$ of the 38 patients with CIDP (table 4). For motor nerve conduction study results, nine $(90 \%)$ of the 10 patients with the distal pattern, and five $(33 \%)$ of the 15 patients with the diffuse pattern had AMNSSR. None of the 13 patients with the intermediate pattern had this pattern of sensory involvement. This pattern of sensory nerve involvement was significantly associated with a monophasic course and long lasting remission.

\section{DISCUSSION}

Our results suggest that patients with CIDP have several patterns of distribution of demyelinative lesions along the course of a nerve, which were categorised to the "distal", "intermediate", or "diffuse" pattern in this study, and that these distribution patterns correlate with clinical features, response to treatment, and outcomes. The high concordance rate of the electrodiagnostic classification between results of the median and ulnar nerve studies (table l) suggest the possibility of a particular predilection of the demyelinative lesions in each patient with CIDP. Moreover, findings of terminal latency index measurement and sensory nerve conduction study support the hypothesis. In this study, proximal regions could not be adequately examined using stimulation at proximal portions or F wave analysis, because of high threshold of the patients' nerves or absent $\mathrm{F}$ waves. Therefore, there may be other distribution patterns of demyelinative lesions such as "predominantly proximal" pattern, if the proximal nerve segments were accurately assessed.

Previous studies showed some factors affecting the clinical features and response to treatment in CIDP. The presence of monoclonal gammopathy or antimyelin associated glycoprotein antibody have been suggested to correlate with some features such as older age of onset, a more indolent course, less severe functional impairment, sensory dominant symmetric polyneuropathy, and a smaller degree of improvement after immunomodulating treatments, ${ }^{6}{ }^{15}{ }^{16}$ and axonal loss has been reported as the major prognostic factor in CIDP. ${ }^{20}$ This study did not include patients with monoclonal gammopathy, and documented a possible association of the patterns of electrodiagnostic findings with clinical profiles.

Distal nerve terminals and nerve roots, where the bloodnerve barrier is anatomically deficient, ${ }^{21}$ are preferentially involved in immune mediated neuropathies such as GuillainBarré syndrome ${ }^{22-24}$ and, probably, CIDP. Dyck et al ${ }^{12}$ showed significant increases in amplitudes of distally evoked compound muscle action potentials in patients with CIDP after successful treatment with plasmapheresis or intravenous immunoglobulin: because the effects were rapid and large, it was likely that resolution of conduction block in the distal nerve segments was the mechanism for improvement. The "distal" pattern in this study was characterised by a marked increase in distal latencies with slowed but relatively preserved nerve conduction velocity, and with no conduction block/abnormal temporal dispersion in the intermediate nerve segments. As shown in fig $1 \mathrm{~A}$, some patients with the distal pattern also showed slowed forearm conduction velocity and slight changes in amplitude and duration of compound muscle action potentials between the wrist and elbow. These findings could reflect demyelination in the forearm (intermediate) segments. However, the disproportionally prolonged distal latency, which was supported by a significantly smaller terminal latency index, suggests preferential demyelination in the distal nerve terminals. Very frequent $(90 \%)$ association of the AMNSSR shown in sensory studies further supports distal predominant demyelination.

Patients with the diffuse pattern showed, besides prolonged distal latencies, profound slowing of nerve conduction, or conduction block in the intermediate nerve segments. We speculate that the diffuse pattern is a severe and advanced form of the distal pattern associated with breakdown of the blood-nerve barrier and, therefore, with involvement of the intermediate segments. Firstly, the two patient groups had common features such as subacute onset, symmetric polyneuropathy, weakness involving proximal as well as distal muscles, and frequent responsiveness to cortcosteroid treatment. Secondly, 33\% of this subgroup of patients also showed AMNSSR, suggestive of distal predominat demyelination in these patients.

During the active phase of some patients with CIDP, nerve biopsy shows endoneurial inflammatory changes with $\mathrm{T}$ lymphocyte infiltration and macrophage mediated 
demyelination. ${ }^{25}$ Both T cells and macrophages secrete TNF- $\alpha$, a proinflammatory cytokine that has toxic effects on peripheral myelin and endothelial cells. ${ }^{26}{ }^{27}$ Increased serum TNF- $\alpha$ has been reported in patients with Guillain-Barré syndrome, and the levels correlate with clinical and electrophysiological severity, suggesting that TNF- $\alpha$ plays a part in the breakdown of the blood-nerve barrier as well as nerve demyelination. ${ }^{28}{ }^{29}$ Our results showed that serum concentrations of TNF- $\alpha$ increased only in patients with the diffuse pattern, suggesting impairment of the blood-nerve barrier in this subgroup of patients.

Patients with the diffuse pattern often expressed responsiveness to immune treatments but often had a treatment dependent relapsing course. These features may be explained by longer disease activity in this subgroup of patients. For both distal and diffuse patient subgroups, it is reasonable that demyelination in the distal nerve terminals and nerve roots is associated with symmetric symptoms and weakness in the proximal as well as distal muscles because nerve length plays little or no part. The extent of increase in CSF protein concentrations, which could reflect breakdown of the blood-CSF barrier surrounding the nerve roots, was more prominent in the "distal" and "diffuse" subgroup patients than the intermediate subgroup patients. Preferential involvement of the nerve terminals and roots in these subgroups suggest that humoral factors such as immunoglobulins, cytokines, and complements may be important for the pathogenesis.

Patients with the intermediate pattern were characterised by a chronic course, asymmetric symptoms, less severe neurological disabilities, and refractoriness to treatments. Despite the asymmetry, all four limbs were involved in most of the patients. It is likely that multiple mononeuropathy constitutes this asymmetric polyneuropathy in these patients, because their conduction abnormalities were distributed multifocally in the intermediate nerve segments as shown in this study. The criteria for CIDP by the American Academy of Neurology do not require symmetric deficits. It is reasonable to include these patients in CIDP, because their condition can respond to immunomodulating treatments. However, their clinical features and treatment responses seem somewhat different from those of patients with symmetric CIDP. This subgroup has been termed multifocal demyelinating sensory and motor neuropathy, and is considered as a multiple mononeuropathy variant of CIDP. ${ }^{78}$ Relative refractoriness to treatments in this subgroup could be due to axonal loss during the long course of the illness.

This study showed that patients with the "distal" motor pattern and AMNSSR had a better response to corticosteroid treatment and showed a monophasic remitting course (table 4 ). It is reasonable that the AMNSSR pattern is associated with better outcome because normal sural sensory responses suggest less axonal loss. Differentiating this subgroup of patients may be important because they are obviously steroid responsive. Intravenous immunoglobulin or plasmapheresis is becoming a common first line therapy for CIDP. Given the side effects profile of immunoglobulin or plasmapheresis compared with that of the long term use of corticosteroids, many clinicians might not regard steroids as the first line therapy for CIDP. However, immunoglobulin therapy and plasmapheresis are expensive or require special equipment. Steroid administration is an inexpensive and readily available alternative. It may be proper to treat patients with demyelination restricted in the distal nerve segments with corticosteroids first.

Because our study was retrospective and uncontrolled, we should be cautious about making assertions about responsiveness to treatments and prognosis. However, clinical and electrophysiological profiles are likely to be distinct among each subgroup. We suggest that CIDP consists of subtypes with varying predilection for lesions along the course of the nerves, as suggested in Guillain-Barré syndrome, ${ }^{22}{ }^{30}$ and that distribution patterns of nerve conduction abnormalities may be useful to predict the responsiveness to treatment and the prognoses of patients with CIDP.

\section{Authors' affiliations}

S Kuwabara, K Ogawara, S Misawa, M Mori, T Hattori, Department of Neurology, Chiba University School of Medicine, 1-8-1 Inohana, Chuo-ku, Chiba 260-8670, Japan

\section{REFERENCES}

1 Ad Hoc Subcommittee for the American Academy of Neurology AIDS Task Force. Research criteria for chronic inflammatory demyelinating polyneuropathy (CIDP). Neurology 1991;41:617-18.

2 Barohn RJ, Kissel JT, Warmolts JR, et al. Chronic inflammatory demyelinating polyradiculoneuropathy. Clinical characteristics, course, and recommendations for diagnostic criteria. Arch Neurol 1989;46:878-84.

3 Hughes R, Sanders E, Hall S, et al. Subacute idiopathic demyelinating polyradiculoneuropathy. Arch Neurol 1992;49:612-16.

4 Dyck PJ, Lais AC, Ohta $M$, et al. Chronic inflammatory demyelinating polyradiculoneuropathy. Mayo Clin Proc 1975;50:62 1-37.

5 McCombe PA, Pollard JD, Mcleod JG. Chronic inflammatory demyelinating polyradiculoneuropathy. Brain 1987;1 10:1617-30.

6 Katz JS, Saperstein DS, Gronseth G, et al. Distal acquired demyelinating symmetric neuropathy. Neurology 2000;54:615-20.

7 Lewis RA, Sumner AJ, Brown AJ, et al. Multifocal demyelinating neuropathy with persistent conduction block. Neurology 1982;32:958-64.

8 Saperstein DS, Amato AA, Wolfe Gl, et al. Multifocal acquired demyelinative sensory and motor neuropathy: the Lewis-Sumner syndrome. Muscle Nerve 1999;22:560-6.

9 Midroni G, Dyck PJ. Chronic inflammatory demyelinating polyradiculoneuropathy. Unusual clinical features and therapeutic responses. Neurology 1996;46:1206-12

10 Thomas PK, Claus D, Jaspert A, et al. Focal upper limb demyelinating neuropathy. Brain 1996;119:765-74.

11 Dyck PJ, Prineas J, Pollard JD. Chronic inflammatory demyelinating polyradiculoneuropathy. In: Dyck PJ, Thomas PK, Griffin JW, et al, eds. Peripheral neuropathy. 3rd ed. Philadelphia: WB Saunders, 1993:1498-517.

12 Dyck PJ, Litchy WJ, Kratz KM et al. A plasma exchange versus immune globulin infusion trial in chronic inflammatory demyelinating polyradiculoneuropathy. Ann Neurol 1994;36:838-45.

13 Pestronk A, Cornblath DR, llyas AA, et al. A treatable multifocal motor neuropathy with antibodies to GM1 ganglioside. Ann Neurol 1988;24:73-8.

14 Chaudhry V, Corse AM, Cornblath DR, et al. Multifocal motor neuropathy: response to human immune globulin. Ann Neurol 1993;33:237-42.

15 Simmons Z, Albers JW, Bromberg MB, et al. Presentation and initial clinical course in patients with chronic inflammatory demyelinating polyradiculoneuropathy: comparison of patients without and with monoclonal gammopathy. Neurology 19993;43:2202-9.

16 Simmons Z, Albers JW, Bromberg MB, et al. Long term follow up of patients with chronic inflammatory demyelinating polyradiculoneuropathy, without and with monoclonal gammopathy. Brain 1995; 118:359-68.

17 Rhee EK, England JD, Sumner AJ. A computer simulation of conduction block: effects produced by actual block versus interphase camcellation. Ann Neurol 1990;28:146-56.

18 Shahani BT, Young RR, Potts F, et al. Terminal latency index (TLI) and late response studies in motor neuron disease (MND), peripheral neuropathies and entrapment syndromes [abstract]. Acta Neurol Scand 1979;73(suppl): 118.

19 Bromberg MB, Albers JW. Patterns of sensory nerve conduction abnormalities in demyelinating and axonal peripheral nerve disorders. Muscle Nerve 1993;16:262-6

20 Bouchard C, Lacroix C, Plante V, et al. Clinicopathologic findings and prognosisi of chronic inflammatory demyelinating polyneuropathy. Neurology 1999;52:498-503.

21 Olsson Y. Topographical differences in the vascular permeability of the peripheral nervous system. Acta Neuropathol 1968;10:26-33.

22 Brown WF, Snow R. Patterns and severity of conduction abnormalities in Guillain-Barré syndrome. J Neurol Neurosurg Psychiatry 1994;54:768-74.

23 Kuwabara S, Yuki N, Koga M, et al. lgG anti-GM1 antibody is associated with reversible conduction failure and axonal degeneration in Guillain-Barré syndrome. Ann Neurol 1998;44:202-8.

24 Kuwabara S, Mori M, Ogawara K, et al. Axonal involvement at the common entrapment sites in Guillain-Barré syndrome with lgG anti-GM1 antibody. Muscle Nerve 1999;22:840-5.

25 Schmidt B, Toyka KV, Kiefer R, et al. Inflammatory infiltrates in sural nerve biopsies in Guillain-Barré syndrome and chronic inflammatory demyelinating polyneuropathy. Muscle Nerve 1996;19:474-87.

26 Uncini A, Di Muzio A, Di Guglielmo G, et al. Effect of rhTNF- $\alpha$ injection into rat sciatic nerve. J Neuroimmunol 1999;95:88-94. 
27 Hall SM, Redford EJ, Smith KJ. Tumor necrosis factor- $\alpha$ has few morphological effects within the dorsal columns of the spinal cord, in contrast to its effects in the peripheral nervous system. J Neuroimmuno 2000; 106:130-6.

28 Sharief MK, McLean B, Thompson EJ. Elevated serum levels of tumor necrosis factor- $\alpha$ in Guillain-Barré syndrome. Ann Neurol 1993;33:591-6.
29 Sharief MK, Ingram DA, Swash M. Circulating tumor necrosis factor- $\alpha$ correlates with electrodiagnostic abnormalities in Guillain-Barré syndrome. Ann Neurol 1997;42:68-73

30 van der Meché FG, Meulstee J, Vermeulen M, et al. Patterns of conduction failure in the Guillain-Barré syndrome. Brain $1988: 111: 405-16$

\section{NEUROLOGICAL PICTURE}

\section{Footdrop after peroneal nerve lesion}

A

45 year old man presented with a history of footdrop. Years before examination he had noticed difficulties with pronation and mild difficulties with elevation of the right toe. He then presented with a 14 day history of acute complete loss of power of foot elevation and pronation and severe paresis of toe extension. Supination and plantar flexion of the foot and toes were intact. Nerve conduction studies showed an axonal lesion of the peroneal nerve. Needle EMG showed acute denervation and reduced interference pattern of the anterior tibial muscle. The long peroneal muscle showed an increase of tissue resistance to needle insertion compatible with muscle fibrolipomatosis. Moreover, there was absence of voluntary muscle activity and infrequent pathological spontaneous activity in this muscle. Magnetic resonance imaging of the lower leg disclosed a fatty degeneration of the long peroneal muscle (fig l A, arrows) on Tl weighted images with only a few residual muscle fibres but with a regular circumference of the muscle. The remaining muscles of the lower leg appeared morphologically intact. On fat suppressed T2 weighted images (fig l B) the anterior tibial muscle and the extensor digitorum muscle (arrows) showed an increased signal consistent with acute denervation. ${ }^{1}$ Thus, the diagnosis of a chronic lesion of the superficial ramus of the peroneal nerve associated with an acute denervation of the deep ramus was confirmed by MRI.

M Bendszus

Department of Neuroradiology, University of Würzburg, Josef-Schneider-Str 11, D-97080 Würzburg, Germany

M Koltzenburg Department of Neurology, University of Würzburg

Correspondence to: $\operatorname{Dr} M$ Bendszus; bendszus@neuroradiologie.uni-weurzburg.de

\section{REFERENCES}

1 Uetani M, Hayashi K, Matsunaga N, et al. Denervated skeletal muscle: MR imaging. Radiology 1993;189:511-15.
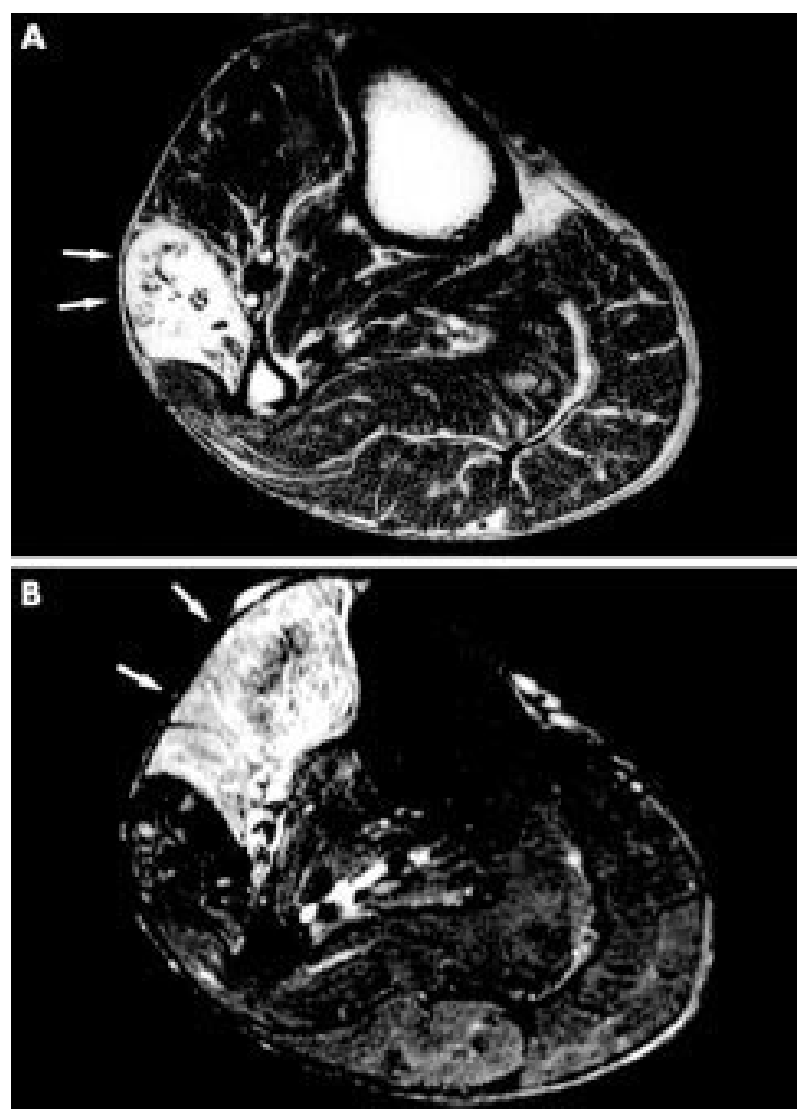